\title{
Influência da estrutura do hábitat sobre a ictiofauna de um riacho em uma micro-bacia de pastagem, São Paulo, Brasil
}

\author{
Cristiane de P. Ferreira \& Lilian Casatti
}

Laboratório de Ictiologia, Departamento de Zoologia e Botânica, Universidade Estadual Paulista. Rua Cristóvão Colombo 2265, 15054-000 São José do Rio Preto, São Paulo, Brasil. E-mail: cristianebio@gmail.com; Icasatti@ibilce.unesp.br

\begin{abstract}
Habitat structure and stream fishes in a pasture basin, São Paulo, Brazil. Four stream stretches (sites 1-4) in a pasture area of the northwestern São Paulo state were studied, aiming to examine the composition and quantitative structure of the ichthyofauna and investigate its relation with habitat features. Fishes were collected during the three most representative months of the dry and wet periods by one electrofishing pass in each month. Chemical water quality analysis showed good conditions in all sites, whereas physical habitat integrity analysis showed poor conditions to the sites 2 and 3 and fair to the sites 1 and 4 . Twenty seven fish species were collected for a total of 1241 specimens and 5,1 kg. The quantitative structure of the ichthyofauna revealed closer association with habitat structure than to seasonality because sites 1 and 2 were split from sites 3 and 4, independently of the season. According to the canonical correspondence analysis, the higher abundance of Gymnotus carapo Linnaeus, 1758 in the sites 1 and 2 was associated to marginal vegetation abundance, the abundance of Piabina argentea Reinhardt, 1867 in the site 3 was explained by the habitat volume, and the abundance of Hypostomus nigromaculatus (Schubart, 1964) in the site 4 was related to the rocky substrate predominance.
\end{abstract}

KEY WORDS. Agroecosystem; mesohabitat; siltation; upper Rio Paraná.

RESUMO. Este trabalho foi desenvolvido em quatro trechos de um riacho em área de pastagem no noroeste do Estado de São Paulo, com o objetivo de examinar a composição e estrutura quantitativa da ictiofauna e investigar sua relação com as características do hábitat. Os peixes foram coletados nos três meses centrais dos períodos seco e chuvoso por meio de uma passagem de pesca elétrica por mês. A análise da qualidade química da água mostrou condições boas nos quatro trechos, enquanto que a análise da integridade física do hábitat mostrou condições pobres para os trechos 2 e 3 e regulares para os trechos 1 e 4 . Foram coletadas 27 espécies de peixes, totalizando 1.241 indivíduos e 5,1 kg. A estrutura quantitativa da ictiofauna demonstrou maior associação com a estrutura do hábitat do que com a sazonalidade visto que os trechos 1 e 2 foram agrupados separadamente dos trechos 3 e 4, independentemente do período de amostragem. De acordo com a análise de correspondência canônica, a elevada abundância de Gymnotus carapo Linnaeus, 1758 nos trechos 1 e 2 esteve associada principalmente com a abundância de vegetação marginal. $O$ maior volume de hábitat no trecho 3 explicou a abundância de Piabina argentea Reinhardt, 1867, e a predominância de substratos rochosos no trecho 4 explicaram a abundância de Hypostomus nigromaculatus (Schubart, 1964).

PALAVRAS-CHAVE. Agroecossistema; Alto Rio Paraná; assoreamento; meso-hábitats.

Dentre os fatores que criticamente afetam a qualidade de ambientes lóticos (Allan \& Flecker 1993) para a ictiofauna, destaca-se a perda e transformação do hábitat interno que geralmente está associada à supressão da vegetação ripária. Como conseqüências ocorrem aumento da incidência de luz, indisponibilização de determinados itens alimentares (p. ex., frutos, sementes e insetos alóctones) e assoreamento dos cursos d'água (Joly et al. 2001). A conversão de florestas nativas em pastagens é tida como um impacto relativamente menos perturbador ao ambiente aquático do ponto de vista químico, uma vez que pastagens não sofrem nenhum tipo de adubação ou aplicações de pesticidas. No entanto, com relação ao carregamento de partículas, alguns autores (YANKeY et al. 1991 apud WOHL \& CARLINe 1996) consideram que pastagens são mais prejudiciais aos ambientes aquáticos que áreas cultivadas, pois contribuem significativamente para o assoreamento dos cursos d'água. Acredita-se, no entanto, que isso seja real apenas para culturas perenes, mas não para culturas anuais, nas quais o solo é revolvido constantemente e se torna facilmente lavável pela chuva.

Revista Brasileira de Zoologia 23 (3): 642-651, setembro, 2006 
Em bacias hidrográficas de pequena escala é comum o registro de focos de assoreamento e enriquecimento nutricional dos corpos d'água pela entrada de fósforo e nitrogênio causados pela presença do gado (Neill et al. 2001).

Embora exista uma série de estudos procurando explicar relações entre elementos da paisagem e uso do solo com a ictiofauna em micro-bacias de regiões de clima temperado (Ward 1989, Roth et al. 1996, Fitzgerald 1998, Lammert \& Allan 1999), na região Neotropical esse tema ainda é pouco explorado. Mesmo nas áreas relativamente mais estudadas, os elementos disponíveis para se investigar tais correlações são insuficientes e esparsos, o que é agravado pelo fato de que $40 \%$ da ictiofauna Neotropical ainda é desconhecida (ReIs et al. 2003). Além do conhecimento taxonômico, o conhecimento da estrutura quantitativa e as relações da ictiofauna com o ambiente também são fundamentais para subsidiar estratégias de conservação e recuperação ambiental. No presente estudo foi determinada a composição e a estrutura quantitativa da ictiofauna de um riacho em área de pastagem no sudeste do Brasil e quais características do hábitat foram as mais relevantes na estruturação das ictiocenoses.

\section{MATERIAL E MÉTODOS}

\section{Área de estudo}

O riacho estudado é o Córrego da Água Limpa, um afluente da margem esquerda do Rio São José dos Dourados, uma das principais bacias de drenagem da região noroeste do estado de São Paulo (Fig. 1). Dentro do seu curso de aproximadamente $16 \mathrm{~km}$ até o reservatório de água que serve como fonte de abastecimento para a cidade de Monte Aprazível, foram selecionados quatro trechos eqüidistantes (T1-T4), de hierarquia fluvial crescente no gradiente longitudinal. É importante ressaltar que à jusante da cidade de Monte Aprazível está instalada uma indústria de beneficiamento de couro, sendo o cromo um dos principais resíduos que pode ser retido pelo solo, representando um problema ambiental crítico (SURITA et al. 2003).

A micro-bacia estudada insere-se na Formação São José do Rio Preto, pertencente ao Grupo Bauru (Iрт 2000). Sua área total é de 7.384 ha, dos quais $64 \%$ são utilizados para pastagem (Alexandre M. Silva, UNESP Sorocaba, comunicação pessoal). Quanto à susceptibilidade à erosão (VIEIRA 1988), 59\% da área da microbacia é considerada muito susceptível (Alexandre M. Silva, comunicação pessoal). O clima da região é tropical quente (NIMER 1989), com duas estações bem definidas, uma chuvosa e quente, de outubro a março, e outra seca e menos quente, de abril a setembro (BARCha \& ARID 1971). A localização e os descritores fisiográficos de cada trecho são apresentados na tabela I.

\section{Métodos}

Para cada trecho de $60 \mathrm{~m}$ de extensão foi realizada amostragem em três viagens à campo na período seco (julho, agosto e setembro de 2004) e na período chuvoso (janeiro, fevereiro e março de 2005), totalizando seis coletas. Em cada coleta foram

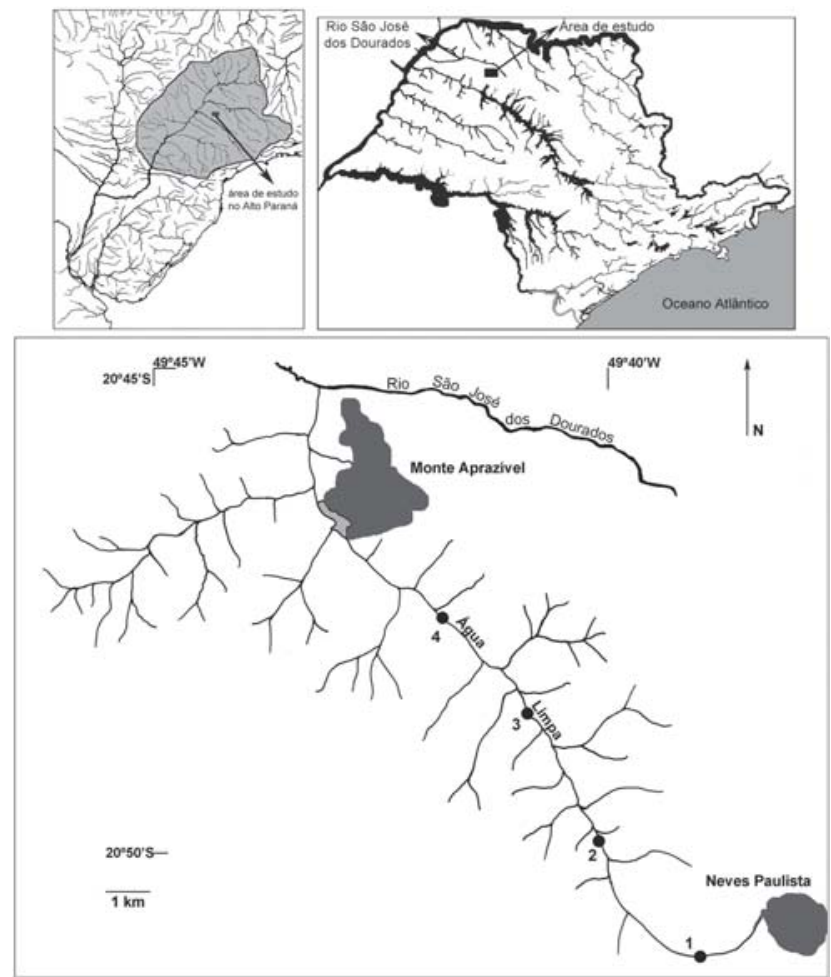

Figura 1. Localização da área de estudo no sistema do Alto Rio Paraná e no Estado de São Paulo, sudeste do Brasil (acima) e os trechos estudados (1-4) ao longo do Córrego da Água Limpa, mostrando sua proximidade com as cidades de Neves Paulista e Monte Aprazível (abaixo).

mensurados sete descritores físico-químicos da água em cada trecho (T1-T4): oxigênio dissolvido, condutividade, $\mathrm{pH}$, turbidez, temperatura, com equipamento eletrônico Horiba ${ }^{\circledR}$, modelo U-10, além do registro de odores distintos, óleo e/ou espuma na água. Nitrato, amônia e ortofosfato foram analisados em laboratório especializado, a partir de amostras de água coletadas no pico dos períodos seco e chuvoso (agosto de 2004 e fevereiro de 2005).

Para a análise física e estrutural do ambiente, a extensão de cada um dos trechos foi subdividida em seis secções transversais, nas quais cinco descritores estruturais do hábitat foram avaliados. A correnteza foi avaliada no meio do canal em cada transecto, com um fluxômetro mecânico General Oceanics ${ }^{\circledR}$, modelo 2030 , por 30 segundos, com três réplicas. A profundidade foi mensurada em cinco pontos eqüidistantes em cada transecto. O tipo de substrato foi avaliado em cinco pontos eqüidistantes em cada transecto, sendo visualmente categorizado em (1) silte, (2) areia, (3) cascalho, (4) seixos, (5) rochas, (6) laje. A abundância de vegetação marginal foi avaliada ao longo das margens de cada transecto, sendo categorizada em (1) ausente, (2) presente, (3) abundante. O volume de hábitat

Revista Brasileira de Zoologia 23 (3): 642-651, setembro, 2006 
Tabela I. Coordenadas, altitude, ordem do trecho e descritores fisiográficos de cada trecho do Córrego da Água Limpa.

\begin{tabular}{|c|c|c|c|c|}
\hline Parâmetros & Trecho 1 & Trecho 2 & Trecho 3 & Trecho 4 \\
\hline Coordenadas & $\begin{array}{c}20^{\circ} 51^{\prime} 08,5^{\prime \prime S} \\
49^{\circ} 39^{\prime} 21,7 " \mathrm{~W}\end{array}$ & $\begin{array}{c}20^{\circ} 50^{\prime} 00,3^{\prime \prime S} \\
49^{\circ} 40^{\prime} 07,5^{\prime \prime} \mathrm{W}\end{array}$ & $\begin{array}{c}20^{\circ} 48^{\prime} 50,4^{\prime \prime S} \\
49^{\circ} 40^{\prime} 41,7 " \mathrm{~W}\end{array}$ & $\begin{array}{c}20^{\circ} 48^{\prime} 06,0^{\prime \prime S} \\
49^{\circ} 41^{\prime} 25,2^{\prime \prime} \mathrm{W}\end{array}$ \\
\hline Altitude (m) & 500 & 490 & 489 & 470 \\
\hline $\begin{array}{l}\text { Ordem do trecho (escala } \\
1: 50.000 \text { ) }\end{array}$ & 1 & 2 & 3 & 4 \\
\hline Vegetação ripária & ausente & ausente & reduzida $(2 \mathrm{~m})$ e alterada & ausente \\
\hline $\begin{array}{l}\text { Vegetação marginal em } \\
\text { contato com a água }\end{array}$ & Poaceae & Typhaceae e Poaceae & ausente & Poaceae e Cyperaceae \\
\hline Meso-hábitats dominantes & poço, corredor e corredeira & corredor & corredor & corredeira e poço \\
\hline Substrato predominante & areia e laje & areia & areia & laje, rocha e cascalho \\
\hline Troncos e galhos & ausente & ausente & raro & ausente \\
\hline
\end{tabular}

foi obtido através da média de todas as profundidades multiplicada pela área de cada trecho.

Seguindo protocolo proposto por CASATTI et al. (2006), cada trecho foi classificado em quatro categorias de integridade físico-química da água (bom, regular, pobre e muito pobre) através do SWI ("stream water index"), e de integridade física do hábitat através do PHI ("physical habitat index"). Cada uma das categorias propostas indica o desvio em comparação com a condição referência para a região (CASATTI et al. 2006).

Para a coleta dos peixes, cada trecho foi bloqueado nos limites jusante e montante e uma passagem de pesca elétrica foi realizada, de acordo com metodologia modificada a partir de Mazzoni et al. (2000) e CAstro et al. (2003). Os indivíduos capturados foram fixados em solução de formalina a $10 \%$ e após 36 horas, em média, transferidos para solução de etanol a $70 \%$. Espécimes testemunhos foram depositados na coleção ictiológica (DZSJRP) do Departamento de Zoologia e Botânica, Universidade Estadual Paulista, São José do Rio Preto, Brasil.

A riqueza de espécies foi estimada com auxílio do programa computacional EstimateS 7 (Colwell 2004), através do estimador ICE (Incidence-based Coverage Estimator), que calcula o fator de correção usando a incidência das espécies raras (aquelas encontradas em até 10 amostragens, LeE \& CHAO 1994), e também através do estimador ACE (Abundance-based Coverage Estimator), cujo fator de correção utiliza a abundância das espécies com até 10 indivíduos nas amostras (Снао et al. 1993).

Como forma de avaliar os dois componentes da diversidade (riqueza e uniformidade), foram calculadas as curvas do componente dominância da diversidade, também chamada de curva de importância de espécies (PianKa 1988), no programa BioDiversity Pro (McAleEce et al. 1997). A diversidade beta, calculada como forma de avaliar a proporção de substituição de espécies ao longo do gradiente longitudinal, foi obtida por meio do coeficiente de similaridade de Jaccard (MagurRan 1988). Os testes estatísticos univariados foram processados utilizando o programa de computador BioEstat 3.0 (Ayres et al. 2003).
A estruturação quantitativa da comunidade foi investigada através da análise de escalonamento multidimensional não métrica (NMDS), uma técnica de ordenação que pode ser aplicada a várias situações ecológicas, cujo cálculo é baseado em uma matriz de dissimilaridade ou de similaridade (Clarke \& WarWicK 1994). O coeficiente de similaridade de Bray-Curtis foi aplicado para a matriz de abundância relativa de cada espécie encontrada em cada trecho a cada amostragem, transformada em $[\log (\mathrm{x}+1)]$ no programa estatístico PRIMER (CLARKE \& GORLEY 2001). A NMDS calcula a distorção, ou stress, entre a matriz de similaridade e a ordenação produzida na representação gráfica dos eixos. No presente estudo, o valor de stress encontrado situa-se em torno de 0,1, o que corresponde a uma boa ordenação, garantindo boa confiabilidade na interpretação dos resultados (CLARKE \& WARWICK 1994).

A relação entre os cinco descritores físicos do hábitat e a estrutura quantitativa da ictiofauna de cada trecho foi avaliada através da análise de correspondência canônica (CCA), implementada no programa computacional CANOCO 4.5 (TER BRAaK \& SMilauer 2002). A CCA permite representar simultaneamente a ordenação espacial de amostras, espécies e variáveis ambientais, que mostram como o ótimo da comunidade varia de acordo com o ambiente (Ter BraAk \& SMilauer 2002). Nesta análise a matriz de abundância de espécies foi transformada $[\ln (A y+B)]$, pesagem diferencial foi atribuída para amostras mas não para espécies. A estabilidade e o significado da CCA foram avaliados através do teste de Monte Carlo, um teste não paramétrico que permite a validação da variância encontrada frente à variância simulada em 5999 permutações.

\section{RESULTADOS}

\section{Características do hábitat}

Dentre todos os descritores físico-químicos analisados (Tab. II), a variação mais expressiva entre os períodos seco e chuvoso ocorreu para o oxigênio dissolvido, cuja concentra- 
Tabela II. Descritores físico-químicos (valores médios) e quantitativos do hábitat em cada trecho do Córrego da Água Limpa, amostrados durante os períodos seco e chuvoso. * Valor de uma amostra.

\begin{tabular}{|c|c|c|c|c|c|c|c|c|}
\hline \multirow{2}{*}{ Descritores } & \multicolumn{2}{|c|}{ Trecho 1} & \multicolumn{2}{|c|}{ Trecho 2} & \multicolumn{2}{|c|}{ Trecho 3} & \multicolumn{2}{|c|}{ Trecho 4} \\
\hline & Seco & Chuvoso & Seco & Chuvoso & Seco & Chuvoso & Seco & Chuvoso \\
\hline Oxigênio dissolvido (mg/l) & 8,4 & 4,2 & 9,8 & 5,1 & 9,1 & 6,1 & 9,8 & 6,1 \\
\hline $\mathrm{pH}$ & 7,1 & 6,5 & 7,0 & 6,5 & 7,2 & 6,7 & 7,6 & 7,0 \\
\hline Condutividade $(\mu \mathrm{S} / \mathrm{cm})$ & 46,7 & 61,3 & 42,3 & 46,7 & 62,7 & 63,3 & 65,0 & 71,0 \\
\hline Turbidez (NTU) & 2,5 & 6,7 & 7,5 & 27,3 & 17,0 & 22,3 & 8,0 & 25,3 \\
\hline Temperatura $\left({ }^{\circ} \mathrm{C}\right)$ & 17,8 & 25,0 & 19,7 & 25,8 & 20,6 & 26,6 & 21,5 & 27,9 \\
\hline Nitrato $(\mathrm{mg} / \mathrm{l})^{*}$ & 0,53 & 0,5 & 1,0 & 0,8 & 1,2 & 0,9 & 1,0 & 0,8 \\
\hline Amônia $(\mathrm{mg} / \mathrm{l})^{*}$ & 0,016 & $<0,001$ & 0,021 & $<0,001$ & 0,004 & $<0,001$ & 0,011 & $<0,001$ \\
\hline Ortofosfato $(\mathrm{mg} / \mathrm{l})^{\star}$ & $<0,01$ & $<0,01$ & $<0,01$ & $<0,01$ & $<0,01$ & $<0,01$ & $<0,01$ & $<0,01$ \\
\hline Odor & normal & normal & normal & normal & normal & normal & normal & normal \\
\hline Óleo/Espuma & ausente & espuma & espuma & espuma & ausente & espuma & ausente & espuma \\
\hline Profundidade média (m) & 0,3 & 0,4 & 0,4 & 0,5 & 0,6 & 0,5 & 0,3 & 0,4 \\
\hline Variação largura (m) & $0,9-5,6$ & $0,7-6,4$ & $1-3,3$ & $0,5-2,7$ & $2,8-5,3$ & $3,7-5,4$ & $1-3,2$ & $0,9-3,4$ \\
\hline Velocidade média da corrente $(\mathrm{m} / \mathrm{s})$ & 0,3 & 0,4 & 0,5 & 0,6 & 0,1 & 0,1 & 0,7 & 0,7 \\
\hline Volume de hábitat $\left(\mathrm{m}^{3}\right)$ & 51,0 & 83,9 & 52,8 & 66,8 & 297,0 & 943,3 & 38,5 & 136,1 \\
\hline
\end{tabular}

ção foi menor no período chuvoso. A qualidade química da água, avaliada através do SWI, foi boa em ambos os períodos, em todos os trechos amostrados.

Cada trecho apresenta características fisiográficas particulares quanto ao tipo de fundo, composição de meso-hábitats e variáveis hidráulicas (Tabs I e II). Na tabela I são descritos três tipos de meso-hábitats dominantes: corredeira, poço e corredor. O meso-hábitat aqui nomeado por corredor se refere aos termos "runs" e "raceways", caracterizados por possuírem águas relativamente rápidas, mais profundas que as corredeiras, com gradiente menor e não turbulentas (RINCón 1999). Durante o período chuvoso registrou-se aumento da profundidade, largura, velocidade da corrente e volume de hábitat (Tab. II).

Os trechos 1 e 4 apresentaram integridade física do hábitat regular, situando-se entre 75 e 50\% da condição referência (CASATTI et al. 2006), principalmente em razão da alta variabilidade de velocidade e profundidade, da alta diversidade de meso-hábitats no trecho 1 e do baixo grau de deposição de sedimento no trecho 4 . Em contraste, os trechos 2 e 3 apresentaram integridade física do hábitat pobre, situando-se entre 50 e $25 \%$ da condição referência (CASATTI et al. 2006), principalmente devido à baixa estabilidade do substrato e elevado grau de sedimento depositado no fundo.

\section{Ictiofauna}

Foram registradas 27 espécies de peixes no Córrego da Água Limpa, das quais 25 no período seco (duas espécies exclusivas desse período) e 25 no período chuvoso (também com duas espécies exclusivas desse período) o (Tab. III). As estimativas de riqueza usando incidência e abundância das espécies raras indicaram que o número de espécies coletadas ficou próximo ao esperado para o riacho como um todo (Fig. 2). Para cada trecho em particular, as estimativas de riqueza apontaram que esforços adicionais de coleta poderiam ser empregados nos trechos 2, 3 e 4 (Fig. 2).

No total foram coletados 1.241 indivíduos e $5.088,7 \mathrm{~g}$ de biomassa, dos quais 563 indivíduos $(45,4 \%)$ e 2.413,6 g $(47,4 \%)$ foram coletados no período seco e 678 indivíduos $(54,6 \%)$ e $2.675,1 \mathrm{~g}(52,6 \%)$ no período chuvoso e tais diferenças foram consideradas significativas (teste de incidência, $\mathrm{p}=0,001$ ). Analisando a variação em termos de abundância em cada trecho, observou-se que durante o período chuvoso houve aumento significativo nos trechos 1 e 2 ( $p<0,001)$, redução significativa no trecho $3(\mathrm{p}=0,01)$ e redução não significativa no trecho 4 $(p=0,92)$. Quanto à biomassa, as diferenças entre os períodos foram significativas apenas nos trechos 1 e 3 ( $p<0,001$ ).

$\mathrm{O}$ trecho 1 apresentou os maiores valores de diversidade $\mathrm{e}$ de equitabilidade, mostrando baixa dominância, ao contrário dos demais trechos (Tab. III). No trecho 2 registrou-se dominância de Gymnotus carapo Linnaeus, 1758, no trecho 3 de Piabina argentea Reinhardt, 1867 e Poecilia reticulata Peters, 1859 e no trecho 4 de Hypostomus nigromaculatus (Schubart, 1964) e $H$. variipictus (Ihering, 1911) (Tab. III). A curva de importância de espécies para cada trecho (Fig. 3) demonstrou que o trecho 1 se enquadra no modelo log-normal, de curva menos inclinada, indicando número relativamente menor de espécies raras e, os demais, no modelo log-series, caracterizado por um pequeno número de espécies abundantes e muitas espécies raras.

No geral, a diversidade beta aumentou no sentido nascente-foz, sendo que do trecho 1 para o trecho 2 , a taxa de substituição de espécies foi de $33 \%$, do trecho 2 para o trecho 3 e do trecho 3 para o trecho 4 esse valor foi superior a $50 \%$. 

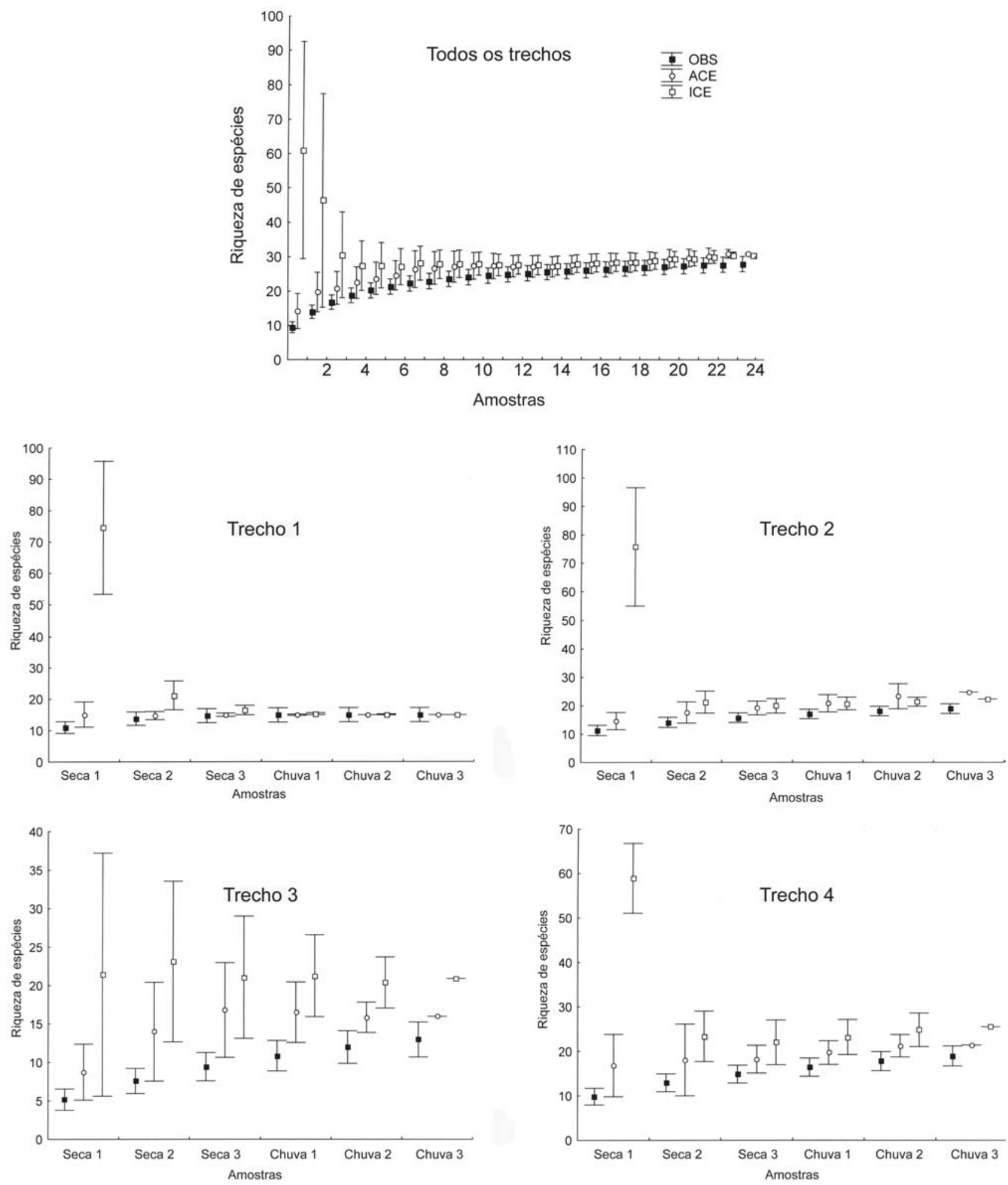

Figura 2. Riqueza de espécies de peixes observada (OBS) e estimadas através do "Abundance-based Coverage Estimator" (ACE) e do "Incidence-based Coverage Estimator" (ICE) para as 24 amostras obtidas (seis amostras em cada trecho).

Revista Brasileira de Zoologia 23 (3): 642-651, setembro, 2006 
Tabela III. Abundância de cada espécie nos períodos seco e chuvoso (com códigos das espécies entre parênteses) e descritores da ictiofauna de cada trecho do Córrego da Água Limpa.

\begin{tabular}{|c|c|c|c|c|c|c|c|c|}
\hline \multirow{2}{*}{ Espécies } & \multicolumn{2}{|c|}{ Trecho 1} & \multicolumn{2}{|c|}{ Trecho 2} & \multicolumn{2}{|c|}{ Trecho 3} & \multicolumn{2}{|c|}{ Trecho 4} \\
\hline & Seco & Chuvoso & Seco & Chuvoso & Seco & Chuvoso & Seco & Chuvoso \\
\hline Apareiodon ibitiensis (Apaibi) & - & - & - & - & - & - & 1 & 1 \\
\hline Apareiodon piracicabae (Apapir) & - & - & - & - & - & - & 1 & - \\
\hline Aspidoras fuscoguttatus (Aspfus) & 11 & 1 & 2 & 6 & - & - & - & - \\
\hline Astyanax altiparanae (Astalt) & 13 & 9 & - & 2 & 4 & 1 & 3 & 2 \\
\hline Callichthys callichthys (Calcal) & 2 & 5 & 2 & - & - & - & - & - \\
\hline Characidium gomesi (Chagom) & - & - & - & - & - & - & 1 & - \\
\hline Cichlasoma paranaense (Cicpar) & - & - & - & 1 & - & - & - & - \\
\hline Corydoras aeneus (Coraen) & 4 & 4 & 20 & 15 & - & - & 1 & 2 \\
\hline Crenicichla britskii (Crebri) & 9 & 11 & 2 & 15 & 3 & - & 6 & 7 \\
\hline Cyphocharax vanderi (Cyphvan) & 6 & - & - & - & - & 1 & 1 & - \\
\hline Eigenmannia virescens (Eigvir) & - & - & 4 & 2 & - & 1 & - & - \\
\hline Geophagus brasiliensis (Geopbra) & - & - & - & - & - & - & 1 & 2 \\
\hline Gymnotus carapo (Gymcar) & 16 & 43 & 55 & 36 & 1 & 1 & 2 & 5 \\
\hline Hisonotus insperatus (Hisins) & - & - & 8 & 13 & - & - & - & - \\
\hline Hoplias malabaricus (Hopmal) & 1 & 3 & 1 & - & - & 1 & - & - \\
\hline Hypostomus ancistroides (Hypanc) & 6 & 13 & 17 & 67 & 1 & 3 & 2 & 15 \\
\hline Hypostomus nigromaculatus (Hypnig) & 10 & 5 & - & - & - & 2 & 109 & 107 \\
\hline Hypostomus variipictus (Hypvar) & - & - & - & - & - & - & 37 & 21 \\
\hline Imparfinis mirini (Impmir) & 9 & 9 & 4 & 38 & - & - & - & - \\
\hline Oligosarcus pintoi (Olipin) & 5 & 45 & 3 & 12 & 2 & - & 5 & 15 \\
\hline Parodon nasus (Parnas) & - & - & - & - & 1 & - & 3 & 5 \\
\hline Phalloceros caudimaculatus (Phacau) & - & - & 6 & 2 & 9 & 11 & - & - \\
\hline Piabina argentea (Piaarg) & 30 & 13 & 15 & 8 & 36 & 11 & 33 & 16 \\
\hline Poecilia reticulata (Poeret) & 6 & 4 & - & 1 & 27 & 23 & - & 2 \\
\hline Rhamdia quelen (Rhaque) & 4 & 1 & 1 & 9 & - & - & - & 1 \\
\hline Serrapinnus notomelas (Sernot) & - & 25 & 1 & 1 & - & - & - & 2 \\
\hline Synbranchus marmoratus (Synbra) & - & - & - & 1 & - & - & - & - \\
\hline Riqueza & 15 & 15 & 15 & 17 & 9 & 10 & 15 & 15 \\
\hline Abundância & 132 & 191 & 141 & 229 & 84 & 55 & 206 & 203 \\
\hline Biomassa (g) & 421,1 & 729,2 & 839,6 & 883,9 & 119,3 & 55,2 & 1033,6 & 1006,8 \\
\hline Riqueza total & \multicolumn{2}{|c|}{16} & \multicolumn{2}{|c|}{19} & \multicolumn{2}{|c|}{13} & \multicolumn{2}{|c|}{18} \\
\hline Índice de diversidade de Shannon-Wiener & \multicolumn{2}{|c|}{1,1} & \multicolumn{2}{|c|}{1,0} & \multicolumn{2}{|c|}{0,7} & \multicolumn{2}{|c|}{0,7} \\
\hline Diversidade teórica máxima & \multicolumn{2}{|c|}{1,2} & \multicolumn{2}{|c|}{1,3} & \multicolumn{2}{|c|}{1,1} & \multicolumn{2}{|c|}{1,3} \\
\hline Equitabilidade & \multicolumn{2}{|c|}{0,9} & \multicolumn{2}{|c|}{0,8} & \multicolumn{2}{|c|}{0,6} & \multicolumn{2}{|c|}{0,6} \\
\hline
\end{tabular}

A estrutura quantitativa da ictiofauna demonstrou maior associação com a estrutura do hábitat do que com a sazonalidade visto que os trechos 1 e 2 foram agrupados separadamente dos trechos 3 e 4, independentemente do período de amostragem (Fig. 4). A CCA mais uma vez reforçou a ordenação produzida na NMDS, identificando quais os descritores de hábitat e espécies foram particularmente relacionados a cada trecho (Fig. 5): a abundância da vegetação marginal nos trechos 1 e 2 esteve associada principalmente à abundância de $G$. carapo, o maior volume de hábitat no trecho 3 explicou a abundância de $P$. argentea e o substrato rochoso no trecho 4 explicou a abundância de $H$. nigromaculatus.

\section{DISCUSSÃO}

\section{Características do hábitat}

O solo dessa micro-bacia é utilizado predominantemente para pastagens, estendendo-se adentro da área de proteção permanente, cuja largura preconizada pelo Código Florestal Brasileiro é de $30 \mathrm{~m}$ em cada margem nos cursos d'água de até $10 \mathrm{~m}$ de largura (Brasil 1989). A ausência de cobertura vegetal e o solo predominantemente arenoso podem ser citados como fatores determinantes no processo de assoreamento desta microbacia, principalmente porque uma expressiva fração da microbacia possui potencial erosivo muito alto. Solos bastante are- 

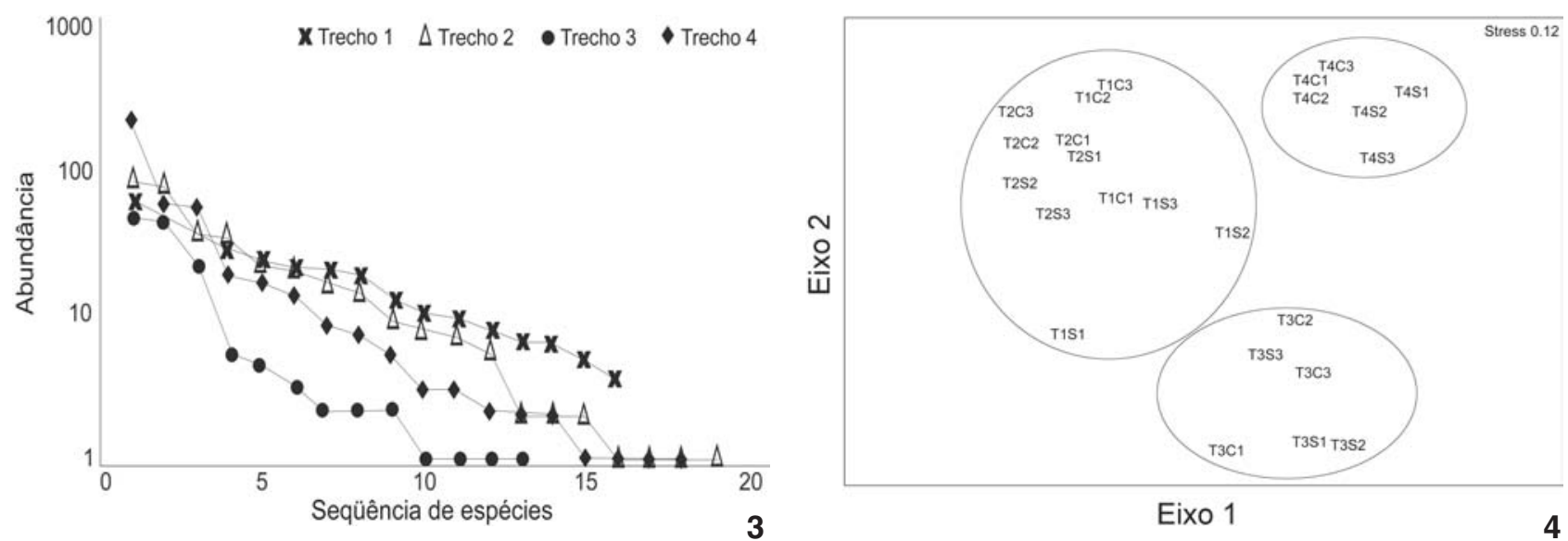

Figuras 3-4. (3) Curva de importância de espécies de cada trecho estudado; (4) análise da estrutura quantitativa das espécies através do método de escalonamento multidimensional não métrico com base na abundância relativa das espécies nos trechos (T1-T4) e nos períodos seco (S1-S3) e chuvoso (C1-C3) no Córrego da Água Limpa, sudeste do Brasil.

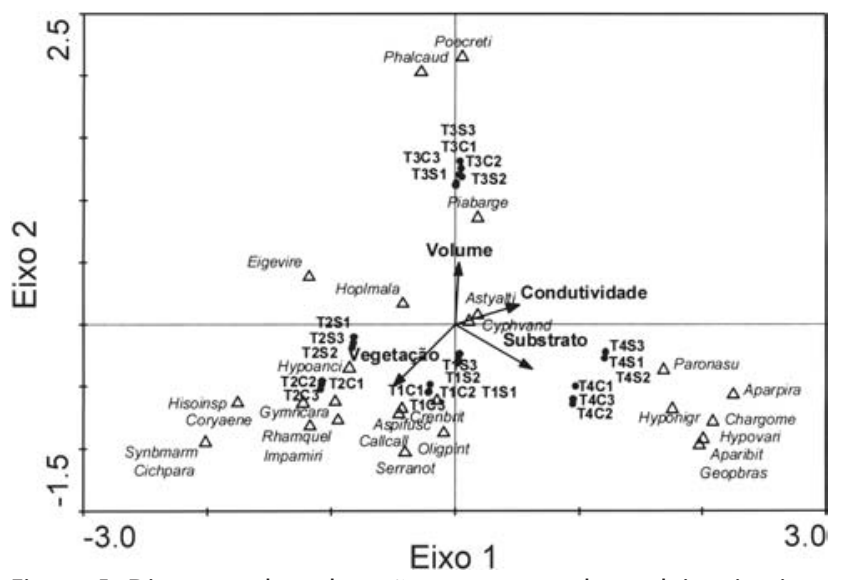

Figura 5. Diagrama de ordenação representando os dois primeiros eixos da análise de correspondência canônica, mostrando os trechos (Trechos 1-4), espécies (triângulos) e descritores ambientais (setas). Veja tabela III para códigos das espécies. Eixos 1 e 2 foram significativos (Teste de Monte Carlo) para trechos e espécies ( $p=$ $0,0002)$

nosos - como o da micro-bacia estudada (veja Ipt 2000) - e em superfícies desprovidas de cobertura vegetal são altamente suscetíveis à erosão por escoamento superficial (CunHa \& GUERRA 1998). Dessa forma, o tipo de cobertura do solo é particularmente importante na prevenção de erosão, pois sob condições naturais toda a formação vegetal (copas das árvores, sub-bosque e serapilheira) atua de forma a amortecer a energia da água vinda da chuva (SiLva et al. 2003).

Não foram encontrados dados históricos com relação a fisiografia e hidráulica da micro-bacia do Córrego da Água Limpa, tornando difícil investigar o grau de assoreamento desse riacho. Entretanto, a julgar pela instabilidade do sedimento, observada principalmente nas porções mais lênticas e pelo grau de erosão nas margens, acredita-se que o carregamento de sedimento nesse curso d'água deva ser de magnitude expressiva, o que reforça ainda mais a importância de projetos de restauração das áreas ripárias da micro-bacia.

Os menores valores de oxigênio dissolvido na água no período chuvoso (veja Tab. II) são explicados pela variação climática sazonal na região e foram registrados por BRANCO \& NecChi JR. (1997), em estudo realizado em três diferentes bacias de drenagem na mesma região. A solubilidade do oxigênio na água está diretamente ligada à variação da temperatura, de modo que quanto maior a temperatura, maior a taxa de desprendimento desse gás para a atmosfera. Da mesma forma, o metabolismo dos organismos aquáticos aumenta em função do aumento da temperatura (Allan 1995).

O riacho estudado se enquadra no padrão para a bacia do rio São José dos Dourados, onde a maior parte dos cursos d'água é desprovida de vegetação ripária (CASATTI et al. 2006). Com a supressão da vegetação ripária e conseqüente aumento da incidência de luz no substrato, a produtividade primária autóctone pode ser incrementada (Pusey \& ARTHINGTON 2003). Ao substituir a base alóctone pela autóctone como fonte energética para a biota aquática, é provável que determinadas espécies sejam excluídas e outras favorecidas. Contudo, na condição de ausência de vegetação ripária, acreditamos que a intensificação do carregamento de partículas leves (areia e silte) para o leito dos riachos dificulte o estabelecimento de organismos fotossintetizantes dependentes de substratos consolidados e estruturalmente mais complexos. Assim, tanto a produção alóctone quanto autóctone fica comprometida em condições de ausência de vegetação ripária em áreas especialmente propensas à erosão do solo, tal como a micro-bacia enfocada neste estudo.

A despeito dos menores valores de oxigênio dissolvido no período chuvoso e elevados valores de amônia no período

Revista Brasileira de Zoologia 23 (3): 642-651, setembro, 2006 
seco, todos os trechos foram considerados bons com relação às características físico-químicas da água, sem variação evidente entre os períodos. Dessa forma, pode-se dizer que não há comprometimento evidente na qualidade da água do córrego estudado para a ictiofauna.

Apesar da ausência de vegetação ripária, os trechos 1 e 4 foram considerados regulares quanto à integridade física, principalmente por apresentarem boa diversidade de hábitats. Em contraste, os trechos 2 e 3 , considerados pobres quanto à integridade física, foram caracterizados pela baixa diversidade de hábitats e pela expressiva sedimentação e instabilidade do substrato, que estão dentre os mais importantes impactos associados à perda de diversidade e integridade das comunidades de peixes (KarR \& SChlosser 1978), sendo muitas vezes mais expressivos do que a perda de qualidade química da água em áreas agrícolas (Wichert \& RAPPORT 1998, CASATTI et al. 2006).

\section{Ictiofauna}

Segundo o conceito de contínuo fluvial (VAnNote et al. 1980), é esperado um aumento gradual na riqueza de espécies ao longo do gradiente longitudinal. Na região tropical, estudos feitos em alguns riachos relativamente íntegros demonstraram a adição de espécies no sentido montante-jusante, acompanhando o incremento da complexidade física e estrutural dos ambientes (Garutti 1988, CasatTI 2005, Braga \& Andrade 2005). Segundo Naiman et al. (1988), no entanto, é difícil aplicar o conceito de contínuo fluvial em sistemas onde existem, naturalmente, zonas bem definidas ou em sistemas que passaram por alterações antropogênicas. No Córrego da Água Limpa, o padrão de incremento longitudinal de espécies não foi detectado. Os altos valores de diversidade beta ao longo do gradiente longitudinal indicam que esse riacho segue, ao invés do padrão típico, uma estruturação por zonas, determinada principalmente pela substituição de espécies (Matrhews 1998).

Alguns resultados do presente estudo refletem a influência da fisiografia do hábitat na estruturação das ictiocenoses. No trecho 3 seria esperado maior riqueza de espécies como resposta ao maior volume de hábitat. Contudo, o volume de hábitat isoladamente nem sempre é capaz de predizer a riqueza de espécies, tal como observado por Angermeier \& Schlosser (1989) em riachos de regiões tropicais e temperadas. Por outro lado, o trecho em questão, apesar de volumoso, possui substrato arenoso, correnteza fraca, conferindo uma situação de baixa complexidade estrutural, o que provavelmente explica a menor riqueza aqui encontrada.

Durante o período chuvoso, registrou-se maior riqueza de espécies, abundância e biomassa geral (Tab. III), corroborando estudos prévios realizados na região Neotropical (GARUTTI 1988, Mazzoni \& Lobón-Cerví́ 2000, Casatti 2005). Tal incremento é geralmente explicado pela intensificação do período reprodutivo e/ou recrutamento de indivíduos de áreas adjacentes (LowEMcConnell 1987) e também pode refletir maior preferência dos peixes por áreas mais volumosas que lhes proporcionam refúgios contra aves e mamíferos predadores (ANGERMEIER \& KARR 1983).
É interessante apontar, contudo, que no trecho 3 o número de indivíduos coletados foi menor no período chuvoso do que no seco e tal diferença parece associada à diminuição da eficiência da amostragem com pesca elétrica, pois as condições hidráulicas do ambiente frente a maiores vazões podem afetar a área de ação do campo elétrico, facilitando o escape dos peixes (ZaLEWski \& Cowx 1990), especialmente em águas mais turvas.

O riacho estudado localiza-se em uma região de relevo relativamente suave, onde acidentes geográficos (p. ex., quedas d'água e cachoeiras) não atuam como agentes isoladores da ictiofauna. Assim, o trânsito das espécies - dadas às limitações intrínsecas de deslocamento de cada espécie - entre os trechos 1 e 4 provavelmente foi pouco afetado por questões fisiográficas. Por outro lado, a ausência de um padrão de adição longitudinal de micro-hábitats e espécies sugere forte relação entre a estrutura física do hábitat e a estrutura da ictiocenose. De fato, através da NMDS (Fig. 4) ficou demonstrado que a estrutura da ictiofauna se organiza mais em função do trecho amostrado do que do período amostral e, através da CCA (Fig. 5), os descritores ambientais associados a cada trecho foram identificados.

No trecho 1, poços, corredores e corredeiras são eqüitativamente representados, disponibilizando hábitats para espécies que exploram a coluna d'água (Astyanax altiparanae Garutti \& Bristki, 2000), fundos arenosos (Aspidoras fuscoguttatus Nijssen \& Isbrücker, 1976 e Corydoras aeneus (Gill, 1858)), vegetação marginal parcialmente submersa (Crenicichla britskii Kullander, 1982 e G. carapo), poços rasos marginais (Serrapinnus notomelas (Eigenmann, 1915) e P. reticulata) e substratos rochosos (H. nigromaculatus), sem dominância expressiva de uma ou outra espécie em particular, com poucas espécies raras e elevada diversidade.

Os demais trechos mostraram-se relativamente mais homogêneos quando comparados ao trecho 1 . O trecho 2 é representado basicamente por um corredor único, com substrato arenoso e margens repletas de gramíneas parcialmente submersas, formando um micro-hábitat particularmente explorado por $G$. carapo, espécie dominante tanto em abundância quanto em biomassa. O trecho 3 é constituído predominantemente por um corredor lêntico volumoso, onde $P$. argentea mostrou-se freqüente e abundante, e raros poços rasos marginais, explorados por $P$. reticulata. No trecho 4 houve predominância de corredeiras de fundos rochosos, exploradas por H. nigromaculatus.

Em síntese, a substituição da vegetação ripária por pastagens e a expansão da área de gramíneas nas margens reduz a diversidade de fluxo de água, confinando-o na região central do canal (Pusey \& ArTHington 2003), além de acarretar mudanças na estrutura do hábitat interno, na qualidade da água e na estrutura das ictiocenoses. Como exemplo disso pode-se citar a expressiva abundância de G. carapo no trecho 2, provavelmente associada à expansão das gramíneas nas margens deste local. Os resultados apontaram forte influência da estrutura física de cada trecho sobre a estrutura das ictiocenoses, sendo que as maiores diversidades específicas foram registradas nos trechos de maior complexidade estrutural.

Revista Brasileira de Zoologia 23 (3): 642-651, setembro, 2006 


\section{AGRADECIMENTOS}

À equipe do Laboratório de Ictiologia DZSJRP-IBILCE pelo auxílio no campo; Francisco Langeani pelo auxílio na identificação taxonômica; Alexandre M. Silva pela análise de uso e ocupação do solo da micro-bacia; Departamento de Zoologia e Botânica e Programa de Pós-Graduação em Biologia Animal IBILCEUNESP pelo apoio; IBAMA pela licença de coleta (001/2003); Mônica C. Bastos, Francisco Langeani, Denise C. Rossa-Feres, Sirlei T. Bennemann e Ricardo M.C. Castro pela leitura crítica do manuscrito. Este trabalho foi subvencionado pela Fundação de Amparo à Pesquisa do Estado de São Paulo (FAPESP) dentro do Programa BIOTA/FAPESP (www.biota.org.br) (01/13340-7, 02/ 05996-2, 03/09612-7).

\section{REFERÊNCIAS BIBLIOGRÁFICAS}

AlLAN, J.D. 1995. Stream ecology. Structure and function of running waters. Dordrecht, Kluwer Academic Publishers, 388p.

Allan, J.D. \& A.S. Flecker. 1993. Biodiversity conservation in running waters. Identifying the major factors that threaten destruction of riverine species and ecosystems. BioScience, Washington, 43 (1): 32-43.

Angermeier, P.L. \& J.R. Karr. 1983. Fish communities along gradients in a system of tropical streams. Environmental Biology of Fishes, Dordrecht, 9: 117-135.

Angermeier, P.L. \& I.J. Schlosser. 1989. Species-area relationships for stream fishes. Ecology, Washington, 70 (5): 1450-1462.

Ayres, M.; M. Ayres Jr.; D.L. Ayres \& A.S. Santos. 2003. BioEstat 3.0: Aplicações estatísticas nas áreas das ciências biológicas e médicas. Belém, Sociedade Civil Mamirauá, 290p.

BARCHA, S.F. \& F.M. ARID. 1971. Estudo de evapotranspiração na região Norte-Ocidental do Estado de São Paulo. Revista Ciências, Votuporanga, 1: 99-122.

Braga, F.M.S. \& P.M. Andrade. 2005. Distribuição de peixes na microbacia do Ribeirão Grande, Serra da Mantiqueira Oriental, São Paulo, Brasil. Iheringia, Série Zoologia, Porto Alegre, 95 (2): 121-126.

BRANCO, L.H.Z. \& O. NECCHI JR. 1997. Variação longitudinal de parâmetros físicos e químicos em três rios pertencentes a diferentes bacias de drenagem na região noroeste do Estado de São Paulo. Acta Limnologica Brasiliensia, São Carlos, 9: 165-177.

Brasil, 1989. Lei $\mathbf{n}^{\mathbf{0}} \mathbf{7 . 8 0 3}$, de 18 de Julho de 1989. Dispõe sobre as alterações da Lei 4.771, que instituiu o Código Florestal Brasileiro. Brasília.

CASATTI, L. 2005. Fish assemblage structure in a first order stream, southeastern Brazil: longitudinal distribution, seasonality, and microhabitat diversity. Biota Neotropica, Campinas, 5 (1): $1-9$.

Casatti, L.; A.M. Silva; F. Langeani \& R.M.C. Castro. 2006. Stream fishes, water and habitat quality in a pasture dominated basin, Southeastern Brazil. Brazilian Journal of Biology, São Carlos, 66 (2b): 681-696.
Castro, R.M.C.; L. Casatti; H.F. Santos; K.M. Ferreira; A.C. Ribeiro; R.C. Benine; G.Z.P. Dargis; A.L.A. Melo; R. Stopiglia; T.X. Abreu; F.A. Bockmann; M. Carvalho; F.Z. Gibran \& F.T.C. Lima. 2003. Estrutura e composição da ictiofauna de riachos do Rio Paranapanema, sudeste do Brasil. Biota Neotropica, Campinas, 3 (1):1-31.

Снао, A.; M.C. Ma \& M.C.K. Yang. 1993. Stopping rules and estimation for recapture debugging with unequal failure rates. Biometrika, London, 80: 193-201.

Clarke, K.R. \& R.M. WARWICK. 1994. Change in marine communities: an approach to statistical analysis and interpretation. Plymouth, Plymouth Marine Laboratory, 144p.

Clarke, K.R. \& R.N. Gorley. 2001. PRIMER v5: User manual/ tutorial. Plymouth, PRIMER-E.

Colwell, R.K. 2004. EstimateS Version 7: Statistical estimation of species richness and shared species from samples [Software user's guide].

Cunha, S.B. \& A.J.T. Guerra. 1998. Geomorfologia do Brasil. Rio de Janeiro, Editora Bertrand, 392p.

Fitzgerald, D.G.; E. Kott; R.P. Lanno \& D.G. Dixon. 1998. A quarter century of change in the fish communities of three small streams modified by anthropogenic activities. Journal of Aquatic Stress and Recovery, New York, 6: 111-127.

GarutTI, V. 1988. Distribuição longitudinal da ictiofauna em um córrego da região noroeste do Estado de São Paulo, bacia do Rio Paraná. Revista Brasileira de Biologia, Rio de Janeiro, 48 (4): 747-759.

IPT. 2000. Diagnóstico da situação atual dos Recursos Hídricos e estabelecimento de diretrizes técnicas para a elaboração do plano da Bacia Hidrográfica do São José dos Dourados. São Paulo, Fundo Estadual de Recursos Hídricos, Instituto de Pesquisas Tecnológicas do estado de São Paulo, Relatório $\mathrm{n}^{\circ} 40675,119 \mathrm{p}$.

Joly, C.A.; J.R. Spigolon; S.A. Lieberg; S.M. de Salis; M.P.M. Aidar; J.P.W. Metzger; C.S. Ziскel; P.C. Lobo; M.T. Shimabukuro; M.C.M. Marques \& A. Salino. 2001. Projeto Jacaré-Pepira O desenvolvimento de um modelo de recomposição da mata ciliar com base na florística regional, p. 271-287. In: R.R. Rodrigues \& H.F. LeITÃo-FilHo (Eds). Matas ciliares: conservação e recuperação. São Paulo, Edusp, 320p.

KARR, J.R \& I.J. SCHLOSSER. 1978. Water resources and the landwater interface. Science, Saint Louis, 201: 229-234.

Lammert, M. \& J.D. Allan. 1999. Assessing biotic biontegrity of streams: effects of scale in measuring the influence of land use/cover and habitat structure on fish and macroinvertebrates. Environmental Management, New York, 23 (2): 257-270.

LeE, S.M. \& A. Chaо. 1994. Estimating population size via sample coverage for closed capture-recapture models. Biometrics, Arlington, 50: 88-97.

Lowe-McConnelL, R.H. 1987. Ecologial studies in tropical fish communities. Cambridge, Cambridge University Press, $382 p$.

Revista Brasileira de Zoologia 23 (3): 642-651, setembro, 2006 
Mazzoni, R. \& J. Lobón-Cerviá. 2000. Longitudinal structure, density and production rates of a neotropical stream fish assemblage: the river Ubatiba in the Serra do Mar, southeast Brazil. Ecography, Lund, 23: 588-602.

Mazzoni, R.; N. Fenerich-Verani \& E.P. Caramaschi. 2000. Electrofishing as a sampling technique for coastal stream fish populations and communities in the southeast of Brazil. Revista Brasileira de Biologia, Rio de Janeiro, 60: 205-216.

Magurran, A.E. 1988. Ecological diversity and its measurement. London, Chapman \& Hall, 178p.

McAleece, N.; P.J.D. Lambshead; G.L.J. Paterson \& J.G. Gage. 1997. Biodiversity professional. Beta-Version. London, The Natural History Museum and the Scottish Association for Marine Sciences.

Matthews, W.J. 1998. Patterns in freshwater fish ecology. London, Chapman \& Hall, 756p.

Naiman, R.J.; H. Décamps; J. Pastor \& C.A. Johnston. 1988. The potential importance of boundaries to fluvial ecosystems. Journal of the North American Benthological Society, Lawrence, 7: 289-306.

Neill, C.; L.A. Deegan; S.M. Thomas \& C.C. Cerri. 2001. Deforestation for pasture alters nitrogen and phosphorus in small amazonian streams. Ecological Applications, Washington, 11 (6): 1817-1828

Nimer, E. 1989. Climatologia do Brasil. Rio de Janeiro, IBGE, 421p.

PIANKA, E. 1988. Evolutionary Ecology. New York, Editora Harper Collins, 356p.

Pusey, B.J. \& A.H. Arthington. 2003. Importance of the riparian zone to the conservation and management of freshwater fish: a review. Marine and Freshwater Research, Collingwood, 54: 1-16.

Reis, R.E.; S.O. Kullander \& C.J. Ferraris Jr. 2003. Check list of the freshwaterfishes of South and Central America. Porto Alegre, Edipucrs, 742p.

Rincón, P.A. 1999. Uso do micro-hábitat em peixes de riachos: métodos e perspectivas, p. 23-90. In: E.P.CARAMASCHI; R. MAZzONI
\& P.R. Peres-Neto (Eds). Ecologia de peixes de riachos. Rio de Janeiro, PPGE-UFRJ, Série Oecologia Brasiliensis, vol. 6, 260p. Roth, N.E.; J.D. Allan \& D.L. Ericson. 1996. Landscape influence on stream biotic integrity assessed at multiple spatial scales. Landscape Ecology, New York, 11 (3): 141-156.

Silva, A.M.; H.E. Schulz \& P.B. Camargo. 2003. Erosão e hidrossedimentologia em bacias hidrográficas. São Carlos, Editora RiMa, 140p.

Surita, C.A.; M.M. Saito \& N. Ellert. 2003. Comportamento do $\mathrm{Cr}, \mathrm{Fe}, \mathrm{Mn}$ e $\mathrm{Al}$ em zona não saturada, em local contaminado por resíduos de indústria de couro no município de Monte Aprazível, São Paulo. Revista Brasileira de Recursos Hídricos, Porto Alegre, 8 (3): 47-58.

Ter Braak, C.J.F. \& P. Smilauer. 2002. CANOCO Reference manual and CanoDraw for Windows user's guide: Software for Canonical Community Ordination (version 4.5). Ithaca, Microcomputer Power, 500p.

Vannote, R.L.; G.W. Minshall; K.W. Cummins; J.R. Sedell \& C.E. CusHING. 1980. The river continuum concept. Canadian Journal of Fisheries and Aquatic Sciences, Ottawa, 37: 130-137.

VIEIRA, L.S. 1988. Manual da ciência do solo: com ênfase nos solos tropicais. São Paulo, Editora Agronômica Ceres, 464p.

WARD, J.V. 1989. The four-dimension nature of lotic ecosystems. Journal of the North American Benthological Society, Lawrence, 8: 2-8.

Wichert, G.A. \& D.J. RAPPORT. 1998. Fish community structure as a measure of degradation and rehabilitation of riparian systems in an agricultural drainage basin. Environmental Management, New York, 22 (3): 425-443.

Wohl, N.E. \& R.F. CARline. 1996. Relations among riparian grazing, sediment loads, macroinvertebrates, and fishes in three central Pennsylvania streams. Canadian Journal of Fisheries And Aquatic Sciences, Ottawa, 53: 260-266.

Zalewski, M. \& I.G. Cowx. 1990. Factors affecting the efficiency of electric fishing, p. 89-111. In: I.G. Cowx \& P. LAMARQUE (Eds). Fish with electricity: Applications in freshwater fisheries management. Oxford, Fishing New Books, 243p.

Recebido em 19.IX.2005; aceito em 09.VIII.2006. 